\title{
Primary Hyperparathyroidism as a Secondary Cause of Depression
}

Katherine Hurst, MSc, MD

A 27-year-old woman was initially diagnosed and treated for depression with suicide ideation. Thirteen months later, kidney stones, an elevated parathyroid hormone, and elevated calcium levels led to a diagnosis of primary hyperparathyroidism. The patient was treated for hyperparathyroidism by resection of the superior right parathyroid gland. When the calcium levels were regulated, the patient's moods, concentration, and memory were back to baseline. (J Am Board Fam Med 2010;23:677-680.)

Keywords: Hyperparathyroidism, Depression, Hypercalcemia, Endocrinology, Clinical Medicine, Biological Markers, Genetics, Nutrition, Vitamin D Deficiency, Case Reports

Primary hyperparathyroidism (PHPT) is an endocrine disease that occurs when the parathyroid glands lose the ability to regulate parathyroid hormone (PTH) secretion via a negative feedback mechanism with calcium. PHPT occurs in 112 of every 100,000 persons. ${ }^{1}$ PHPT occurs with highest incidence in the sixth and seventh decade of life $^{2}$; the average age at diagnosis is 55 years. ${ }^{3}$ PHPT reaches a prevalence of $2 \%$ to $3 \%$ among postmenopausal women. ${ }^{4}$ Eighty percent to $90 \%$ of cases are caused by a single enlarged parathyroid gland. ${ }^{2}$

PHPT is the leading cause of elevated calcium among patients presenting in the primary care setting. ${ }^{5}$ The symptoms of PHPT include fatigue, weakness, kidney stones, depression, decreased bone mineral density, abdominal pain, and anxiety. PHPT can also present with no signs or symptoms. Up to $80 \%$ of patients are asymptomatic and are diagnosed only after routine laboratory tests show elevated calcium levels. ${ }^{6}$

\section{Case Report}

On October 3, 2006, a 27-year-old Hispanic woman presented with a 2 -year history of trouble

This article was externally peer reviewed.

Submitted 14 August 2009; revised 18 February 2010; accepted 1 March 2010.

From Cedar Rapids Medical Education Foundation and Linn Community Care, Cedar Rapids, IA.

Funding: none.

Conflict of interest: none declared.

Corresponding author: Katherine Hurst, MSc, MD, Linn Community Care, 1201 Third Avenue SE, Cedar Rapids, IA 52403 (E-mail: KatherineLHurst@aol.com). with memory, violent mood swings, thoughts of death, trouble concentrating, and suicidal ideation. The patient gave examples of driving and not knowing where she going or why, forgetting lunch dates with family members, instigating arguments with her husband, and feeling that life was so miserable she would be better off dead. The patient's medical history included one term pregnancy and no current medications. Her family history was negative for mental illness and positive for kidney stones in paternal relatives. The patient was married, had a young child, and was a full-time student at a local community college. She did not use tobacco products, alcohol, or drugs. The patient was initially treated with citalopram $20 \mathrm{mg}$ (1 tablet by mouth daily) and lorazepam $0.5 \mathrm{mg}$ (by mouth every 8 hours as needed) for anxiety.

On October 10, 2006, there was little change in the patient's symptoms. Citalopram was increased to $40 \mathrm{mg}$ by mouth daily. Fourteen days later the patient complained of dry mouth and decreased energy and libido. Lorazepam was discontinued and bupropion was added at a dose of $150 \mathrm{mg}$ by mouth every 24 hours. At the next appointment, on December 21, 2006, the patient admitted to not taking the bupropion. After refusing previous referrals to a psychiatrist, the patient accepted at this time; however, she did not go. She also missed her next clinic appointment, which had been scheduled for December 29, 2006. 
On February 3, 2008, the patient presented at an emergency room with right abdominal pain. An abdominal computed tomography scan showed multiple left intrarenal calculi, right hydronephrosis, and a 5-mm calculus in the uretovesicular junction. Laboratory evaluation revealed a calcium level of $12.5 \mathrm{mg} / \mathrm{dL}$ (normal range, $8.5-10.2 \mathrm{mg} / \mathrm{dL}$ ) (Table 1). The patient was told she had kidney stones. She was prescribed hydrocodone $5 \mathrm{mg}$ and acetaminophen $500 \mathrm{mg}$ and was advised of the need for hydration and to make an appointment with her primary care physician.

On February 8, 2008, tests to measure PTH and calcium levels were ordered. The PTH was 200 $\mathrm{pg} / \mathrm{mL}$ (normal range, $10-65 \mathrm{pg} / \mathrm{mL}$ ) and her calcium level was $11.7 \mathrm{mg} / \mathrm{dL}$. The combination of persistent elevated serum calcium and PTH met the requirement for a diagnosis of PHPT. A referral to an endocrinologist was made.

On the first visit to endocrinology on June 10, 2008 the PTH was $248.5 \mathrm{pg} / \mathrm{mL}$, alkaline phosphate was $138 \mathrm{U} / \mathrm{L}$, and calcium was $12.9 \mathrm{mg} / \mathrm{dL}$. The patient met the National Institute of Health's criteria for parathyroidectomy by being younger than 50 and having neuropsychiatric symptoms as well as nephrolithiasis.

A Sestamibi scan showed a parathyroid adenoma of the right superior parathyroid gland. The right superior parathyroid gland was removed on July 7 , 2008. Intraoperative PTH monitoring was done (see Table 2); baseline PTH level was $341.1 \mathrm{pg} /$ $\mathrm{mL}$. Ten minutes after the adenoma was removed, the PTH level was $54.8 \mathrm{pg} / \mathrm{mL}$. Twenty minutes later the PTH level was $27.5 \mathrm{pg} / \mathrm{mL}$. The pathology report diagnosis was hypercellular parathyroid. Approximately 1 month after surgery the patient's moods and concentration were much improved.

On September 11, 2008, the laboratory values were as follows: calcium level, $9.3 \mathrm{mg} / \mathrm{dL}$; phosphate level, $3.2 \mathrm{mg} / \mathrm{dL}$; PTH level, $81.8 \mathrm{pg} / \mathrm{mL}$. The endocrinologist ordered a test to measure the patient's 25 hydroxy vitamin D level because of an elevated PTH level with a normal calcium level. It was low at $24 \mathrm{mg} / \mathrm{mL}$. The endocrinologist postulated that the elevated PTH level was caused by vitamin $\mathrm{D}$ deficiency. The patient was prescribed 50,000 units of ergocalciferol by mouth every week for 12 weeks, then every other week.

On June 23, 2009, the patient had a follow-up appointment with her endocrinologist. She admitted to initially being noncompliant with the pre- scribed vitamin D supplementation. At this visit her calcium level was $9 \mathrm{mg} / \mathrm{mL}$, her PTH level was 75 $\mathrm{pg} / \mathrm{mL}$, and her 25 hydroxy vitamin D level was 18 $\mathrm{ng} / \mathrm{mL}$. She was treated with 12 more weeks of ergocalciferol 50,000 units, then 12 weeks of the same medication every other week.

The most recent laboratory findings came from a tests drawn on January 8, 2010. Her calcium level was $10 \mathrm{mg} / \mathrm{dL}$, her PTH level was $67 \mathrm{pg} / \mathrm{mL}$, and her 25 hydroxy vitamin D level was $23 \mathrm{ng} / \mathrm{mL}$. The patient continues to supplement with 50,000 units ergocalciferol every other week and has her calcium, PTH, and vitamin D levels monitored every 6 months.

\section{Literature Review}

A PubMed search was done using $\mathrm{MeSH}$ terms "depression" and "PHPT," with limits that included "humans," "English," "adults aged 18 and over," and "written from 1900 to present." A relevant case report from 1960 written by Mandel ${ }^{7}$ was found. A 67-year-old housewife was treated for depression with 8 treatments of electroconvulsive therapy. The patient responded well and was discharged on November 22, 1958. She returned on March 20, 1959, with depression and mental confusion. She was given 8 more electroconvulsive treatments. Incidentally, a urinary tract infection was found via urinalysis. A urogram showed hydronephrosis of the right kidney with a large calculus. Her serum calcium level was $6 \mathrm{Meq} / \mathrm{mL}$ (normal, 4.5 Meq/mL). An exploration of the parathyroid gland found a large adenoma, weighing $5 \mathrm{~g}$, attached to the left inferior parathyroid gland. After removal of the overgrown gland and calcium regulation there was no recurrence of psychotic depression. ${ }^{7}$

The effectiveness of surgery for alleviating depression from PHPT is supported in the literature. In a study of 360 patients who underwent parathyroidectomy for PHPT from August 1994 to September 2002, 35 patients met criteria for major depression. ${ }^{8}$ Of these patients, $37 \%$ required antidepressant medication before surgery. Postoperatively, $90 \%$ stated that depression no longer impaired their ability to work, and 52\% reported improved quality of life. Among the patients taking antidepressants, $27 \%$ of those no longer used medication and $27 \%$ used reduced doses of antidepressant medication. ${ }^{8}$ 


\begin{tabular}{lccccc}
\hline & Albumin (G/dL) & Creatinine (mg/dL) & TSH (UI/mL) & Calcium (mg/dL) & PTH (pg/mL) \\
\hline Normal values (range) & $3.4-4.8$ & $0.7-1.4$ & $0.27-4.2$ & $8.5-10.5$ & $10-65$ \\
Date & & & & & \\
3 Feb 2008 & 4.5 & 0.9 & 12.5 & 11.7 & 200 \\
8 Feb 2008 & 4.5 & 0.62 & 0.44 & 12.9 & 248.9 \\
10 June 2008 & 4.6 & 0.9 & 0.98 & & \\
\hline
\end{tabular}

TSH, thyroid-stimulating hormone; PTH, parathyroid hormone.

Surgical removal of the parathyroid adenoma results in regulation of calcium levels. It remains unknown exactly how calcium level disturbances contribute to mood changes. A study of cerebrospinal fluid (CSF) from patients with PHPT gave some insight. The CSF concentrations of both total and ionized calcium were increased among patients who had PHPT. The increase in ionized calcium in the CSF correlated with a decrease in 5HIAA, a serotonin metabolite. ${ }^{9}$

\section{Discussion}

Common themes in these cases were the symptoms of depression resolving after calcium levels were regulated, the fortuitous finding of elevated calcium levels, and an added element that is out of the norm for clinical depression. Mandel ${ }^{7}$ acknowledged that it had long been recognized that abnormal levels of calcium, both high and low, can lead to psychosis. Mandel's ${ }^{7}$ new finding was a difference in the typical depressive patient and the addition of mental confusion that he observed. In the patient presented here, thoughts of homicide, suicide, mood swings, and forgetfulness were out of character for an initial presentation of clinical de- pression. The patient in this case also expressed her doubt of the diagnosis of depression. She was not compliant with medication because she did not believe the diagnosis, and she simply dealt with her mood swings and thought pattern problems on her own.

$\mathrm{Mandel}^{7}$ stated the importance of excluding chemical causes of depression in the 1960s. The clinical application is obtaining an electrolyte panel, including serum calcium level, as a screening test. Depression has a high prevalence among patients in the primary care setting, consisting of $10 \%$ to $14 \%$ of all reasons for a visit. ${ }^{10,11}$ Because of this high prevalence it is important to thoroughly evaluate physical causes of mental health deterioration.

Secondary causes of depression should be assessed during the initial visit and considered at each follow-up appointment. A review of systems, social history, and family history; physical examination; medicine reconciliation; drug and food allergy updates; and laboratory tests can rule out many secondary causes of depression. Another important point to consider when prescribing medication is a patient's ability to take the medication safely. The liver (more specifically, the cytochrome P450 en-

Table 2. Laboratory Values After Parathyroidectomy

\begin{tabular}{|c|c|c|c|}
\hline & Calcium (mg/dL) & PTH (pg/mL) & 25-Hydroxy Vitamin D (ng/mL) \\
\hline Normal values (range) & $8.5-10.5$ & $10-65$ & $32-100$ \\
\hline \multicolumn{4}{|l|}{ Date } \\
\hline \multicolumn{4}{|l|}{7 July 2008} \\
\hline Before removal of adenoma & & 341.1 & \\
\hline 10 minutes after removal of adenoma & & 54.8 & \\
\hline 20 minutes after removal adenoma & & 27.5 & \\
\hline 29 July 2008 & 9.6 & 44.4 & \\
\hline 11 Sept 2008 & 9.3 & 81.8 & 24 \\
\hline 23 June 2009 & 9.0 & 75 & 18 \\
\hline 8 Jan 2010 & 9.8 & 67 & 23 \\
\hline
\end{tabular}

PTH, parathyroid hormone. 
zyme family) metabolizes most psychiatric medications. Laboratory tests can rule out kidney or liver disease. Potential drug-drug interaction must also be analyzed during each encounter. Depression may occur secondary to medical conditions such as electrolyte disturbance, Cushing syndrome, endocrinologic and neurologic disorders, hypothyroidism, medication use, or withdrawal from medications such as anticholinergics or stimulants. ${ }^{12}$

\section{Conclusion}

Depression is a diagnosis of exclusion. It is important to address secondary causes of depression during each visit, especially when multiple medications have been ineffective or symptoms are out of the ordinary for clinical depression.

\section{References}

1. Mazzaglia PJ, Berber E, Kovach A, Milas M, Esselstyn C, Siperstein AE. The changing presentation of hyperparathyroidism over 3 decades. Arch Surg 2008;143:260-6.

2. Suliburk JW, Perrier ND. Primary hyperparathyroidism. Oncologist 2007;12:644-53.

3. Taniegra ED. Hyperparathyroidism. Am Fam Physician 2004;69:333-9.
4. Jorde R, Bonaa KH, Sundsfjord J. Primary hyperparathyroidism detected in a health screening. The Tromso study. J Clin Epidemiol 2000;53:1164-9.

5. Kearns AE. Primary hyperparathyroidism: is there anything new? Compr Ther 2004;30:105-10.

6. Blackburn M, Diamond T. Primary hyperparathyroidism and familial hyperparathyroid syndromes. Aust Fam Physician 2007;36:1029-33.

7. Mandel MM. Recurrent psychotic depression associated with hypercalcemia and parathyroid adenoma. Am J Psychiatry 1960;117:234-5.

8. Wilhelm SM, Lee J, Prinz RA. Major depression due to primary hyperparathyroidism: a frequent and correctable disorder. Am Surg 2004;70:175-9; discussion, 9-80.

9. Joborn C, Hetta J, Niklasson F, et al. Cerebrospinal fluid calcium, parathyroid hormone, and monoamine and purine metabolites and the blood-brain barrier function in primary hyperparathyroidism. Psychoneuroendocrinology 1991;16:311-22.

10. Leon AC, Olfson M, Broadhead WE, et al. Prevalence of mental disorders in primary care. Implications for screening. Arch Fam Med 1995;4:857-61.

11. Goodwin RD, Kroenke K, Hoven CW, Spitzer RL. Major depression, physical illness, and suicidal ideation in primary care. Psychosom Med 2003;65:501-5.

12. Moore DP, Jefferson JW. Handbook of medical psychiatry. 2nd ed. Philadelphia: Elsevier Mosby; 2004. 